
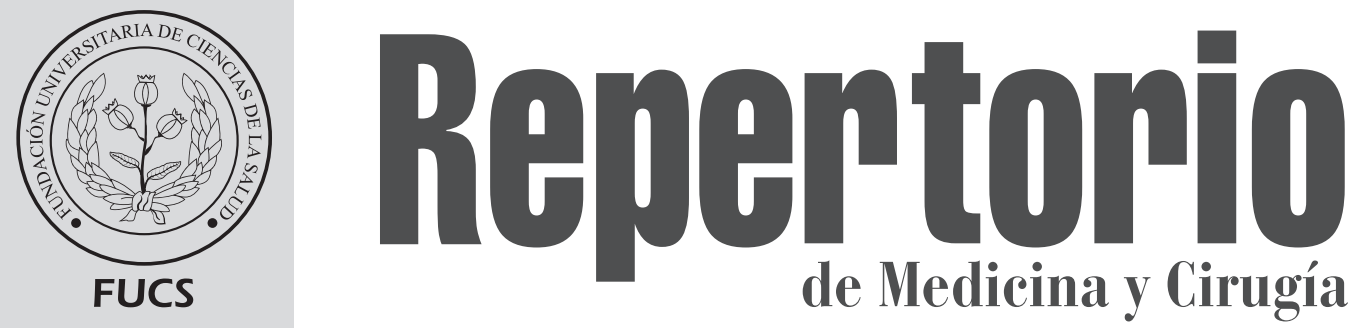

Vol. 29
No2.2020

ISSN: 0121-7372 • ISSN electrónico: 2462-991X

Artículo de revisión

\title{
Derecho al consumo informado: el caso de las bebidas azucaradas en Colombia
}

Diana Rocío Bernal MD, PhD Nancy Milena Bernal MD

\section{Right to informed consumption: the case of sugar-sweetened beverages in Colombia}

${ }^{a}$ Bioética y Biojurídica, Grupo de Investigación en Derechos Humanos. Directora de la Especialización en Derecho Médico-Sanitario, Facultad de Jurisprudencia de la Universidad del Rosario, Bogotá DC, Colombia.

${ }^{b}$ Pediatría, Hospital Infantil Universitario de San José. Bogotá DC, Colombia.

\section{R E S U M EN}

Introducción: se presenta un análisis sobre el derecho al consumo informado en el caso de las bebidas azucaradas, a través del recorrido administrativo y judicial en Colombia. Metodología: se hace desde una perspectiva jurídica, bioética y médica, señalando cómo la protección judicial de este derecho se da gracias al activísimo social y judicial que se enfrenta con el lobby de la industria, cuyos intereses también inciden, no en el ámbito del acceso a la justicia, pero si en el del diseño de políticas públicas en salud para reducir el consumo de bebidas azucaradas. Conclusiones: de la revisión de la literatura se encontró que ciertas enfermedades no transmisibles se asocian con el consumo de estos productos, por lo cual en diferentes regiones del mundo se han replanteado medidas para mitigar su impacto, mientras en Colombia el escenario desde las políticas públicas no ha sido favorable.

Palabras clave: derecho a la información, derecho a la salud, edulcolorantes, obesidad, protección del consumidor.

(C) 2020 Fundación Universitaria de Ciencias de la Salud - FUCS. Este es un artículo Open Access bajo la licencia CC BY-NC-ND (http://creativecommons.org/licenses/by-nc-nd/4.0/).

\section{INFORMACIÓN DEL ARTÍCULO}

Historia del artículo:

Fecha recibido: septiembre 4 de 2019

Fecha aceptado: febrero 11 de 2020
Autor para correspondencia.

Dra. Diana Rocio Bernal

diana.bernalc@urosario.edu.co
DOI

10.31260/RepertMedCir.01217273.967 
Introduction: an analysis of the right to informed sugar-sweetened beverages consumption is herein presented from the administrative and legal background in Colombia. Methodology: a study conducted from the legal, bioethical and medical perspective, pointing out how legal protection of this right is possible thanks to social and legal activism which faces industrial lobby whose interests do not influence the access to justice institutions but does influence the design of health public policy for reducing sugar-sweetened beverages intake. Conclusions: our review of the literature found that certain non-communicable diseases are associated with the intake of these products for which measures to mitigate their impact have been reconsidered in different regions of the world, while in Colombia the public policy scenario has been unfavorable.

Key words: right to information, right to health, artificial sweeteners, obesity, consumer protection.

(C) 2020 Fundación Universitaria de Ciencias de la Salud - FUCS. This is an open access article under the CC BY-NC-ND license (http://creativecommons.org/licenses/by-nc-nd/4.0/).

\section{INTRODUCCIÓN}

El consumo de bebidas azucaradas ha aumentado en el mundo a lo largo de las últimas décadas. Algunos estudios dan cuenta de su incremento en varias zonas de América Latina y el Caribe, donde se ha expandido en forma considerable tanto el de las comerciales como de bebidas caseras azucaradas ${ }^{1}$ y su asociación con problemas de salud. Así, se ha reportado que la ingesta de azúcares libres, entre ellos los contenidos en productos como las bebidas azucaradas y en general de alimentos densamente calóricos que se caracterizan por su bajo contenido nutricional, en contraste con los altos niveles de grasas saturadas, ácidos grasos trans, azúcares libres y/o sal, solos o en combinación, con una actividad física insuficiente, constituyen un factor estrechamente relacionado con el incremento de las tasas actuales de obesidad y diabetes en el mundo, así como del riesgo de T2DM y de enfermedades coronarias. ${ }^{2-4}$ Incluso, se han asociado con este consumo altas tasas de mortalidad en ciertas regiones. Singh y col. evidencian que Latinoamérica y el Caribe "tienen la mayor mortalidad absoluta relacionada con el consumo de bebidas azucaradas-SSB- (48 000 por millón de adultos, 95\% de IU, 41 000-54 000)", en donde "casi 1 de cada 10 DALY relacionados con la diabetes mellitus" se asociaron con el consumo de SSB $(9.7 \%, 95 \%$ UI, $4 \%-18.3 \%)^{\prime \prime}$. $^{5}$

El sobrepeso y la obesidad conllevan a múltiples comorbilidades e incluso se les atribuye una mortalidad de 3,4 millones de defunciones anuales, y se relacionan con $44 \%$ de mellitus tipo 2, 23\% de las cardiopatías isquémicas y entre $7 \%$ y $41 \%$ con algunos tipos de cáncer ${ }^{6}$

La prevalencia de sobrepeso en niños menores de cinco años en 2012 aumentó a 30 millones en países en desarrollo y en los desarrollados esa cifra fue de 10 millones. A nivel mundial $10 \%$ de los niños en edad escolar presentan sobrepeso. El autor menciona que en América Latina se identificó que entre 22,2 a 25,9 millones de niños en edad escolar tienen exceso de peso; de estos 34,5\% pertenecen a México, 33,5\% a Brasil y 18,9\% a Colombia. ${ }^{6}$

En este sentido diversos estudios dan cuenta de la necesidad de adelantar diferentes intervenciones que incluyen educación, cambios de estilos de vida y como además lo recomienda la OMS, la necesidad de una regulación sobre las bebidas azucaradas y los alimentos ultraprocesados, que pueden constituirse en estrategias políticas efectivas con incidencia directa e indirecta en la promoción de alimentación saludable. ${ }^{7}$

\section{ESTRATEGIA METODOLÓGICA}

El diseño de investigación responde a un estudio exploratorio con el fin de examinar el contexto de la pregunta de investigación planteada ${ }^{8}$, que se desarrolla desde tres perspectivas metodológicas: la jurídica-analítica, la revisión de literatura y el análisis bioético de casos. Para este fin se aplicó el método documental o bibliográfico ${ }^{9}$ que incluyó la revisión de casos jurídicos administrativos y judiciales de normativa nacional e internacional, así como de revisión de literatura jurídica y médica sobre la materia, que combina los lineamientos básicos de la revisión bibliográfica descriptiva y los casos clínicos con revisión. ${ }^{10-12}$ El método de revisión del caso clínico se adaptó al estudio sobre el consumo de bebidas azucaradas en Colombia a partir del movimiento desarrollado por educar consumidores y RedPaPaz, documentado en sus páginas web, en medios de comunicación (prensa, radio y televisión) y redes sociales (facebook y twitter). La revisión de literatura se hizo a partir de los tesauros seleccionados de Unesco y DeSC, y su revisión en las bases de datos Pubmed, Scopus, Scielo, Vlex y Redalyc.

Para la revisión del caso desde la perspectiva bioética, se hizo un análisis a partir de los principios bioéticos de la Declaración Universal de Bioética y Derechos Humanos. ${ }^{13}$ 


\section{Caso}

En 2016, la organización no gubernamental (ONG) Educar Consumidores emitió un comercial de aproximadamente 30 segundos, que buscaba impulsar el debate sobre la necesidad de disminuir el consumo de bebidas azucaradas. Como respuesta a esta medida publicitaria una de las empresas de bebidas azucaradas más grandes del país interpuso una queja ante la Superintendencia de Industria y Comercio (SIC) del país, con el fin de cancelar la divulgación del contenido del mencionado comercial. ${ }^{14,15}$ La SIC resolvió el tema mediante la resolución 59176 de 2016, y con ocasión de dos acciones de tutela ${ }^{i}$ la Corte Constitucional se pronunció sobre el tema mediante la sentencia T-543 de 2017, que reconoció y protegió los derechos de los accionantes. Por su parte, la ONG RedPaPaz lideró la campaña "nocomasmasmentiras" con el fin de promover información relacionada con la comida chatarra así como con los jugos y gaseosas azucaradas. ${ }^{16} \mathrm{Se}$ hizo a través de la producción de piezas comerciales y del sitio web www.nocomasmasmentiras.org. Además, presentó en diciembre de 2017 ante la SIC una queja por publicidad engañosa relacionada con las bebidas Hit de Postobón S.A. y Fruper de Alpina Productos Alimenticios S.A., toda vez que la información relacionada con contenido y valores nutricionales no correspondía a la realidad y sus niveles de azúcar superaban los recomendados por la OMS, aunque la SIC no reconoció a esta ONG como tercero interviniente. ${ }^{16,17}$

En este contexto el grupo de trabajo territorial occidente 1 del INVIMA, en marzo de 2016 adelantó una serie de visitas a Postobón SA en sus instalaciones de la ciudad de Medellín (Antioquia) e impuso la "medida sanitaria de seguridad consistente en suspensión total de los servicios de publicidad en página web". Así mismo, el grupo de trabajo remitió las diligencias a la dirección de responsabilidad sanitaria del Invima, que mediante auto No. 2018001868 ordenó iniciar proceso sancionatorio y formular cargos contra la sociedad Gaseosas Posada Tobón S.A. ${ }^{18}$ Ante la ausencia de una decisión de fondo por parte de estas entidades, RedPaPaz en septiembre de 2018 instauró una acción de tutela solicitando que:

1) se le reconociera como tercero interviniente dentro de los procesos administrativos por publicidad engañosa contra Alpina y Postobón que adelanta la SIC; 2) se ordenara al INVIMA dar respuesta completa a la solicitud de información formulada el 15 de marzo de 2018, en donde solicitaba información sobre las acciones adelantadas contra el producto Hit de Alpina; 3) se ordenara a la SIC informar a Red Papaz sobre las actuaciones adelantadas en las quejas contra Alpina y Postobón; y 4) se ordenara a la SIC dar un trámite prevalente, tomar las medidas cautelares que sean procedentes y adoptar una decisión de fondo con respecto a las denuncias por publicidad engañosa en contra de Alpina y Postobón elevadas el 21 de diciembre de 2017.
Tanto el juez de primera instancia como de segunda denegaron las pretensiones. Como consecuencia la organización RedPaPaz presentó un escrito de insistencia ante la Corte Constitucional, con el fin de que el expediente de tutela T-7139620 fuese seleccionado para su estudio por la honorable corte ${ }^{17-19}$, siendo en efecto seleccionada ${ }^{\mathrm{ii}}$ y contando otra vez, con la intervención favorable de Dejusticia. ${ }^{20}$

Por otro lado, la SIC mediante la resolución 19022 de 31 de mayo de 2019, decidió iniciar investigación administrativa con formulación de cargos contra Gaseosas Posada Tobón S.A. por "presunta vulneración a los derechos de los consumidores a recibir información y productos de calidad, así como por la posible transgresión a las disposiciones sobre calidad, información, condiciones objetivas, publicidad engañosa, propaganda comercial de precios y comparativa" conforme con las disposiciones vigentes de protección de los derechos de los consumidores, ante lo cual la empresa ha señalado que actualmente cumple con la normatividad del INVIMA para la producción de este tipo de bebidas. ${ }^{21}$

Por último, se debe mencionar que en ejercicio del mismo activismo social, las organizaciones lograron el apoyo de varios congresistas para la presentación del proyecto de ley 214 de 2018, "por medio de la cual se promueve el acceso a información necesaria para fomentar entornos alimentarios saludables y prevenir Enfermedades No Transmisibles y se adoptan otras disposiciones". ${ }^{22}$

Este proyecto establecía lineamientos específicos sobre el etiquetado de bebidas azucaradas, con énfasis en los datos y características que debían incluirse en el producto para la toma de decisiones informadas relacionadas con el consumo de este tipo de bebidas. Sin embargo, por la falta de discusión del proyecto en la respectiva legislatura en el Congreso de la República, se archivó en el primer semestre de 2019.

\section{Bebidas azucaradas y salud}

El consumo de bebidas azucaradas se relaciona con obesidad y sobrepeso, los cuales a su vez son predisponentes para enfermedades no transmisibles como cardiovasculares, diabetes y cáncer. Así mismo la obesidad y el sobrepeso son factores de riesgo modificables y la disminución en el consumo de bebidas azucaradas reduce la prevalencia de las enfermedades no transmisibles mencionadas antes. ${ }^{23}$

A lo largo del tiempo estos factores de riesgo han ido en aumento; en 2010 se estimó que el sobrepeso y la obesidad causaban 3,4 millones de muertes en todo el mundo y los costos anuales de atención médica atribuibles a esta condición de salud superaban los \$ 600 mil millones. ${ }^{23}$

Diferentes estudios realizados en el último año sobre el consumo de dichas bebidas y el desarrollo de enfermedades no transmisibles, han pretendido demostrar una relación ya sea directa de causa-efecto o su asociación como factor de

${ }^{i}$ Estas acciones de tutela fueron interpuestas por la Asociación Educar Consumidores, RedPaPaz, Dejusticia y otras organizaciones.

ii A la fecha de presentación de este artículo, el caso no ha sido resuelto por la Corte Constitucional Colombiana. 
riesgo. La publicación en 2019 de un análisis prospectivo en el Jackson Heart Study por asoció como una probabilidad significativa alta el mayor consumo de bebidas azucaradas con el riesgo elevado de enfermedad renal crónica (ERC). ${ }^{24}$ Otro estudio realizado en México, presentó hallazgos relacionando el efecto del consumo de bebidas azucaradas sobre la obesidad en la población adolescente, concluyendo que "el alto consumo de bebidas azucaradas aumentó las probabilidades de aumentar de peso y de circunferencia de cintura". ${ }^{25}$

Existen medidas desde el punto de salud pública que pueden disminuir el consumo de bebidas azucaradas y por ende generar un impacto en la salubridad de la población con disminución en la prevalencia de obesidad y sobrepeso. Esto ha sido ampliamente estudiado y cada vez más expertos, incluida la Organización Mundial de la Salud, promueven intervenciones como el aumento de impuestos indirectos que afecten los precios de las bebidas azucaradas. ${ }^{26}$

\section{Aspectos legales relacionados con el consumo informado de bebidas azucaradas}

Una aproximación jurídica al tema implica tomar en cuenta al menos tres cuestiones fundamentales: primero, los derechos de los consumidores en cuanto a conocer el consumo informado, la libertad de expresión e información y el derecho a la salud; segundo, los impuestos sobre las bebidas azucaradas como medida de salud pública para desincentivar el consumo de éstas en la población; y tercero, las leyes de etiquetado como garantía para los consumidores.

\section{DERECHOS DE LOS CONSUMIDORES}

La publicidad y las reclamaciones realizadas por las organizaciones Educar Consumidores y RedPaPaz estaban enfocadas en la defensa de los derechos de los consumidores de las bebidas azucaradas en Colombia, en especial los niños y niñas adolescentes. Sin embargo, la SIC consideró que precisamente el comercial que emitían estas organizaciones vulneraba el derecho a la información de los consumidores.

A juicio de esta entidad, la información contenida en el comercial no contaba con soporte o evidencia científico o médica que soportara la veracidad de los datos señalados por la ONG en la pieza publicitaria objeto de la discusión. ${ }^{27}$ Por esta razón, la publicidad objeto de cuestionamiento inducía a error a los consumidores al atribuirle efectos nocivos a un producto sin contar con el debido sustento para ello, violando así lo dispuesto en el Estatuto del Consumidor, el cual establece que los consumidores tienen derecho a recibir información consecuente y coherente que les permita tomar decisiones de consumo razonables y de contar con información completa, veraz, transparente, oportuna, verificable, comprensible e idónea respecto de los productos que se ofrezcan en el mercado. ${ }^{27}$
Como consecuencia de esta reclamación la SIC mediante la resolución No. 59176 del 7 de septiembre de 2016, ordenó cesar de manera inmediata la difusión del comercial de televisión que se transmite actualmente relacionado con el consumo de bebidas azucaradas (gaseosas, jugos embotellados y té helado), el cual hace alusión a unas supuestas repercusiones adversas en la salud por el consumo de las mismas. ${ }^{27}$

Con posterioridad a esta resolución, el máximo Tribunal Constitucional mediante la sentencia de tutela No T-543-17 en relación con las cuestiones de fondo sobre la información del comercial, centró el debate en torno a cuatro puntos principales que se desprenden de la interpretación del artículo 20 de la Constitución Política Colombiana. ${ }^{28}$

a. El derecho a la libertad de expresión, que tiene su reconocimiento en instrumentos internacionales como la Declaración Universal de Derechos Humanos, el Pacto Internacional de Derechos Civiles y Políticos o la Convención Americana de Derechos Humanos, y es el pilar fundamental para la coexistencia de sociedades plurales, libres y democráticas.

b. El derecho a la libertad de información, hace parte del derecho a la libertad de expresión y se refiere de manera especial a los derechos relacionados con la búsqueda $\mathrm{y}$ acceso a la información, la libertad de información y el derecho a recibirla, todo enmarcado en los principios de veracidad e imparcialidad.

c. La prohibición de censura, conforme a lineamientos nacionales e internacionales implica la necesidad de limitar diferentes controles o vetos respecto de la información previa su difusión.

d. Las condiciones para limitar las publicaciones en internet, de acuerdo con los estándares nacionales e internacionales sobre la materia, en especial conforme al Comité de Derechos Humanos que ha señalado como las limitaciones que se lleguen a establecer respecto de la información o el funcionamiento de sitios web es permisible siempre que sean compatible con el artículo 19, párrafo 3, del Pacto Internacional de Derechos Civiles y Políticos. Finalmente, la sentencia $\mathrm{T}-543 / 17$ reconoció la vulneración del derecho a la libertad de información por parte de la SIC, mediante la citada resolución No. 59176/16, la cual adoptó medidas no establecidas en la legislación colombiana ni con una finalidad imperiosa o necesaria, y considera que además incurre en una forma de censura previa, en contravención a la Convención Americana sobre Derechos Humanos y por la Constitución Política de Colombia, al imponer un control previo sobre el contenido de la información a transmitir. ${ }^{28}$

El derecho a la libertad de información conexo al del consumo informado, implica que de manera efectiva los consumidores puedan acceder a información adecuada que les permita tomar decisiones según sus necesidades e intereses, de forma que el Estado debe garantizar este derecho que en las reglamentaciones avanzadas como la de la Unión Europea, se refieren a la información y el acceso a la misma para distinguir entre un consumidor medio 
y uno vulnerable. ${ }^{29}$ El vulnerable no tiene la capacidad de acceder o de comprender en forma adecuada, lo cual implica "tomar en consideración sus necesidades especificas y reforzar sus capacidades". ${ }^{30}$ Este concepto vulnerable se aplica al consumidor alimentario que requiere de información detallada y comprensible para garantizar sus derechos, conforme al reglamento 1169/2011 que establece medidas especiales de protección para este tipo de clientes alimentarios. ${ }^{31}$

El derecho a la información se relaciona de manera estrecha con la libertad de expresión relacionada con la publicidad de este tipo de bebidas. La publicidad juega un rol importante en la toma de decisiones sobre el consumo de bebidas azucaradas puesto que se caracteriza por el uso de elementos visuales y slogans para promover que es "cool" consumirlas. ${ }^{32}$ En esta forma, la libertad es una garantía para quienes pretenden brindar información veraz y transparente, pero es un límite para aquellos que, sin información veraz, pretenden inducir las decisiones de los consumidores.

\section{LOS IMPUESTOS A LAS BEBIDAS AZUCARADAS}

Con fundamento en estos datos que dan cuenta de las altas tasas de obesidad y diabetes, muchos países han implementado diferentes estrategias nacionales para la promoción de estilos de vida saludables y la prevención de la obesidad. ${ }^{33}$ Estas recomendaciones han incluido actividad física y dietas con alimentos y bebidas bajas en calorías y libres de azúcares, sobre todo en el caso de los niños, adolescentes y adultos jóvenes, tomando como punto de partida la estrategia mundial sobre régimen alimentario, actividad física y salud de la OMS. ${ }^{34}$

En el caso del consumo de bebidas azucaradas, muchos países han optado por establecer impuestos siguiendo la recomendación de la OMS, que indica que las políticas fiscales de los países deberían favorecer los productos alimenticios y las bebidas saludables. Conforme a los informes de esta organización, las medidas tributarias pueden generar impactos positivos sustanciales en la salud pública. ${ }^{26,35,36}$

Así, en Estados Unidos se aprobó este impuesto en San Francisco, Oakland, Berkeley y Albany en California, Filadelfia (PA), Cook County (IL) y Boulder (CO). El Reino Unido también aprobó un impuesto a las bebidas con más de $5 \mathrm{mg}$ de azúcar, excepto leches y zumos de frutas.

La medida cobra vigencia a partir de abril 2018 y con el dinero recaudado se espera la financiación de actividades deportivas en diferentes colegios. ${ }^{37}$ En el continente europeo países como Portugal, Finlandia, Francia, Dinamarca y la comunidad de Cataluña en España, también han gravado con impuestos alimentos y/o bebidas azucaradas. En la misma línea están países como Sudáfrica, India, Sri Lanka,
Fiji y Hungría, que paulatinamente han gravado estas bebidas. ${ }^{36,38,39}$

En algunos casos se gravan solo los refrescos con azúcar añadida, en otros se incluyen zumos, productos lácteos y bebidas con edulcorantes. Algunos países tasan por igual cualquier líquido con azúcar añadido. En otros aunque no existe este gravamen sobre las bebidas azucaradas, si hay un fuerte activismo, en algunos casos desde las organizaciones no gubernamentales y en otros desde el mismo gobierno, con el fin de lograr que se establezca un impuesto a estas bebidas azucaradas para contribuir a la lucha contra la obesidad y la diabetes. Esta ha sido el caso de Canadá y Australia, en donde diferentes reportes científicos recomiendan esta clase de impuestos como ingresos para financiar iniciativas que beneficien a las personas con mayores desventajas y así impactar en equidad social ${ }^{40}$ y como política pública para reducir la obesidad..$^{36,41,42}$

La región de América Latina y el Caribe, reportada como una de las zonas de mayor consumo de bebidas azucaradas, no ha sido ajena a esta discusión. Dominica y Barbados en el Caribe en 2015 adoptaron un impuesto del 10\%. ${ }^{42}$ En la misma línea, en la zona continental, se encuentran México, Brasil, Chile y Ecuador. Mientras tanto, países como Argentina y Panamá, se encuentran dando la discusión sobre el tema. ${ }^{36,42-44}$

Por su parte, Colombia no ha sido ajeno a la discusión jurídica sobre el impuesto a las bebidas azucaradas. Así, el texto inicial de la propuesta a la reforma tributaria de 2016 establecía el llamado Impuesto nacional al consumo de bebidas azucaradas, que se generaba sobre: 1) bebidas azucaradas, entre las que se incluyen las energizantes, saborizadas y en general cualquiera que contenga azúcares añadidos o edulcorantes; y 2) concentrados, polvos y jarabes que después de su mezcla o dilución, permiten la obtención de bebidas azucaradas, energizantes o saborizadas. El impuesto se fijaba en $\$ 0.1050$ dólares por cada litro (mil centímetros cúbicos o su equivalente)". ${ }^{45}$

Este gravamen fue apoyado por los ministros de hacienda y de salud y protección social, resaltando que estaba dentro del paquete de estrategias del gobierno para la reducción de la obesidad y la promoción de la actividad física y los estilos de vida saludable en la población, lo cual fue respaldado por un informe del ministerio y por la comunidad científica y académica colombiana. ${ }^{45-47}$ Sin embargo, para el primer debate del proyecto ante la comisión tercera del Congreso de la República, ya se había eliminado esta propuesta de impuesto tras un fuerte cabildeo. ${ }^{48,49}$

En la actualidad la llamada ley de financiamiento en relación con las bebidas azucaradas eliminó el IVA monofásico para transformarlo en uno plurifásico, que como bien lo señala el investigador Alejandro Rodríguez Llach ${ }^{50}$, es una medida insuficiente desde la perspectiva de salud pública, en tanto que el precio real de las bebidas no aumenta en forma significativa para efectos de desincentivar su consumo, a diferencia de lo que sucedería con un impuesto exclusivo. 


\section{LEYES SOBRE ETIQUETADOS}

El derecho al consumo informado relacionado con las bebidas azucaradas incluye que estos productos tengan datos detallados y comprensibles en su etiquetado. En el país no existe una ley específica al respecto para esta clase de productos, pero gracias al activismo de diversas organizaciones como Educar Consumidores y RedPaPaz, en los últimos tres años se han presentado algunas propuestas legislativas tendientes a regular el tema. En 2016 se presentó el proyecto conocido como "Ley para el consumo informado de azúcar" ${ }^{\prime \prime}$, que buscaba obligar a los fabricantes para incluir en las etiquetas de bebidas azucaradas advertencias sobre los riesgos para la salud. Sin embargo, este proyecto fue archivado. Un año después, se presentó un nuevo proyecto que buscaba establecer "medidas de salud pública para el control de la obesidad y otras enfermedades crónicas no transmisibles derivadas" ${ }^{\prime \prime}$, y entre sus disposiciones establecía normas específicas sobre el etiquetado de productos comestibles y bebidas de alto contenido calórico y/o bajo valor nutricional. Este proyecto también fue archivado.

Por último, existe un proyecto de la ley 214 de 2018 mediante el cual "se promueve el acceso a información necesaria para fomentar entornos alimentarios saludables y prevenir enfermedades no transmisibles y se adoptan otras disposiciones. [Etiquetado de alimentos en prevención de obesidad]", que, en lo particular, establece una regulación específica de etiquetado para productos comestibles y bebibles procesados y ultraprocesados..$^{53}$

Una medida de etiquetado específico fortalecería el derecho al consumo informado de este tipo de bebidas azucaradas, pues como se reportó en el estudio de Roberto, Wong y col. ${ }^{54}$ existe una alta probabilidad de percepción de los consumidores respecto de los riesgos para la salud de las bebidas azucaradas, lo que puede incidir en la reducción de la compra de los mismos.

\section{Principios bioéticos, derechos humanos y bebidas azucaradas}

La información y la toma de decisiones sobre el consumo de bebidas azucaradas va más allá de los lineamientos jurídicos o recomendaciones médicas, puesto que también conlleva una reflexión bioética. Es importante hacer referencia a la responsabilidad ética que tiene esta industria sobre la salud de la población en general, que no puede concretarse en programa de responsabilidad social empresarial encaminados a la promoción de la actividad física, pues como lo señalan algunos estudios, al final estas medidas se traducen más bien en estrategias de relaciones públicas que de fomento real a estilos de vida saludable. ${ }^{55}$

Para efectos de las políticas de salud pública que deben tomar los estados, se debe partir del hecho de que la alimentación y el consumo de bebidas azucaradas están relacionados con la salud y, en consecuencia, con el derecho al disfrute del más alto nivel posible de salud, como se mencionó en el informe de la relatora Anand Grover. ${ }^{56,57}$ Los estados, con el fin de prevenir los daños a la salud de la población y cumplir las obligaciones dimanantes del derecho a la salud, deben formular politicas nacionales para reglamentar la publicidad de alimentos poco saludables, elaborando leyes y un marco normativo con miras a reducir la exposición de los niños a las potentes técnicas de venta de alimentos y bebidas. Esas reglamentaciones deben garantizar que la industria alimentaria ofrezca información nutricional exacta y fácil de leer en la publicidad de sus productos.

En efecto, dentro del informe de la relatora a los estados parte, se encuentran una serie de recomendaciones relacionadas con medidas que deben tomar los países sobre alimentos poco saludables para la población general y en forma especial en grupos vulnerables, así mismo unas específicas hacia el control de la industria de alimentos. En este sentido, el informe de la relatora recomienda a los estados parte que adopten medidas tendientes a: e) Alentar a las empresas transnacionales, mediante incentivos y otras medidas fiscales, a fabricar y vender alimentos y bebidas más saludables que no sean perjudiciales para la salud; y f) Reglamentar la comercialización, publicidad y promoción de alimentos poco saludables, especialmente la dirigida a las mujeres y los niños, para reducir su visibilidad y aumentar la de alimentos más saludables, exigiendo, por ejemplo, a los supermercados que coloquen las frutas y verduras en lugares más accesibles y visibles. ${ }^{57}$

Así mismo señala: A fin de cumplir su obligación de hacer efectivo el derecho a la alimentación de los grupos vulnerables como los niños, las mujeres y los grupos de población de bajos ingresos, el relator especial recomienda que los estados adopten las siguientes medidas:

a) Abordar la cuestión de los estereotipos de género en la preparación de las comidas que dan un trabajo excesivo a las mujeres;

b) Preparary poner en práctica programas de educación para la salud con objeto de promover la elección de alimentos sanos en marcos institucionales tales como escuelas, centros de salud, centros juveniles y lugares de trabajo, con la participación de los niños, los padres y los empleados, respectivamente;

Reconociendo el papel de la industria alimentaria en la carga creciente de las enfermedades no transmisibles, el Relator Especial recomienda que esta industria adopte las siguientes medidas: a) adoptar directrices nutricionales internacionalmente aceptables y cumplir las directrices promulgadas al respecto en el plano nacional; b) abstenerse de comercializar, promover o hacer publicidad de alimentos poco saludables para la población, especialmente para los niños; c) invertir en la mejora del contenido nutricional de los alimentos poco saludables; d) aumentar la transparencia de la información nutricional sobre los productos alimenticios, y abstenerse de hacer afirmaciones falsas o que se presten a confusión respecto de la salud; e) abstenerse de socavar los esfuerzos de salud pública relacionados con la nutrición, en 
particular mediante recursos tales como la financiación y la publicidad de investigaciones tendenciosas, la creación de grupos de encubrimiento y los litigios largos y onerosos. ${ }^{57}$

Desde esta perspectiva, los principios bioéticos de autonomía, beneficencia, responsabilidad social y salud de la declaración universal de bioética y derechos humanos, aplican especialmente para el desarrollo de políticas públicas encaminadas a fomentar la salud física y mental de las personas. ${ }^{13,58}$

\section{CONCLUSIÓN}

Retos para alcanzar los estándares más altos de salud y nutrición a partir del consumo responsable e informado de bebidas azucaradas

Las regiones con las tasas más altas de obesidad y diabetes, como son América Latina, el Caribe y el caso que nos ocupa, Colombia, no pueden ser ajenas a la adopción de medidas jurídicas y políticas que influyan en la toma de decisiones, siguiendo así las recomendaciones de diferentes organismos internacionales, entre ellos la OMS y diferentes estudios que dan cuenta de la efectividad de medidas políticas como los impuestos. $35,36,59,60$

En este sentido los países deben asumir mayores compromisos que complementen el ámbito de la autorregulación y la responsabilidad social corporativa, en este caso de la industria correspondiente y empoderen a los consumidores para la toma de decisiones asertivas. Si bien es importante la información que se suministra en el etiquetado de estos productos, en muchos casos tiene un papel limitado para efectos de esta toma de decisiones, pues existen otros factores "como el marketing, la situación del momento de compra, el precio o el entorno socio-cultural" que inciden directa e indirectamente en la elección de compra del consumidor. ${ }^{61,62}$

Así mismo, el profesional de la salud debe informar a los pacientes los riesgos del consumo de bebidas azucaradas, incluso desde edades muy tempranas para así crear hábitos saludables. Las modificaciones epigenéticas desde el útero incluyen una adecuada ganancia de peso en la mujer gestante, promoción de leche materna exclusiva hasta los 6 meses y complementaria hasta los dos años conforme a las recomendaciones de la OMS, así como no ofrecer sal ni azúcar el primer año de vida y por supuesto evitar el consumo de bebidas azucaradas toda la vida. Así es más fácil prevenir en edades tempranas que en la etapa adulta.

En este sentido diferentes países se han inclinado por establecer impuestos específicos a las bebidas azucaradas. En América Latina fue México el primero y en Colombia la reciente reforma tributaria planteaba tal impuesto, pero no se logró establecer. Como medida adicional existe un fuerte activismo desde sectores sociales, académicos y de salud, para fomentar la necesidad de proporcionar mayor información sobre el consumo de bebidas azucaradas. El reto en este campo, además del análisis juicioso de la necesidad de un impuesto a las bebidas azucaradas, también se encuentra en fortalecer el derecho al consumo informado de la población respecto de estas bebidas, como una medida de salud pública para combatir la epidemia de enfermedades no transmisibles.

\section{CONFLICTOS DE INTERÉS}

Las autoras manifiestan no tener conflictos de interés.

\section{REFEREN CIAS}

1. Singh GM, Micha R, Khatibzadeh S, Shi P, Lim S, Andrews KG, Engell RE, Ezzati M, Mozaffarian D, Global Burden of Diseases Nutrition and Chronic Diseases Expert Group (NutriCoDE. Global, regional, and national consumption of sugar-sweetened beverages, fruit juices, and milk: a systematic assessment of beverage intake in 187 countries. PloS. 2015;10(8): e0124845.

2. Malik VS, Willett WC, Hu FB. Global obesity: trends, risk factors and policy implications. Nat Rev Endocrinol. 2013;9(1):13.

3. Alwan A., Armstrong T., Bettcher D., Branca F., Chisholm D., Ezzati M., Garfield R., MacLean D., Mathers C., Mendis S., Poznyak V. Global status report on noncommunicable diseases: description of the global burden of NCDs, their risk factors and determinants. Ginebra: World Health Organization; 2011.

4. World Health Organization. Fiscal policies for diet and prevention of noncommunicable diseases: technical meeting report, 5-6 May 2015, Geneva, Switzerland: World Health Organization; 2016.

5. Singh GM, Micha R, Khatibzadeh S, Lim S, Ezzati M, Mozaffarian D. Estimated global, regional, and national disease burdens related to sugar-sweetened beverage consumption in 2010. Circulation. 2015;132(8):639-66.

6. Mancipe Navarrete JA, Villamil G, Samanta S, Correa Bautista JE, Meneses-Echávez JF, González-Jiménez E, Schmidt-RioValle J. Efectividad de las intervenciones educativas realizadas en América Latina para la prevención del sobrepeso y obesidad infantil en niños escolares de 6 a 17 años: una revisión sistemática. Nutr. Hosp. 2015;31(1):102-14.

7. Gómez LF, Ibarra ML, Lucumí DI, Arango CM, Parra A, Cadena Y, Erazo V, Parra DC. Alimentación no saludable, inactividad física y obesidad en la población infantil colombiana: un llamado urgente al estado y la sociedad civil para emprender acciones efectivas. Global Health Promotion. 2012;19(3):87-92.

8. Batthyány $\mathrm{K}$, Cabrera M. Metodología de la investigación en Ciencias Sociales. Apuntes para un curso inicial [Methodology of research in Social Sciences. Notes for an initial course]. Montevideo: Universidad de la República; 2011.

9. Botero AB. La metodología documental en la investigación jurídica: alcances y perspectivas. Opinión jurídica. 2003;2(4):109-116.

10. Guirao G, Adolf SJ. Utilidad y tipos de revisión de literatura. Ene. $2015 ; 9(2)$. 
11. Grant MJ, Booth A. A typology of reviews: an analysis of 14 review types and associated methodologies. Health Info Libr J. 2009;26(2):91-108.

12. Icart MT, Canela J. El artículo de revisión. Enferm Clínica. 1994;4(4):180-4.

13. United Nation Educational, Scientific and Cultural Organization. Universal Declaration on Bioethics and Human Rights [Internet]. Paris: Unesco; 2005 [citado 19 Febrero 2019]. Recuperado de http:// portal.unesco.org/es/ev.php-URL_ID=31058\&URL_DO=DO_ TOPIC\&URL_SECTION $=201 . \mathrm{html}$

14. El Espectador. Este es el polémico comercial de bebidas azucaradas que sacaron del aire; 2016. [citado 22 de febrero de 2019]. Disponible en: https://www.elespectador.com/noticias/ salud/el-polemico-comercial-de-bebidas-azucaradas-sacaron-delarticulo-653715.

15. Educar Consumidores. Campaña contra el consumo excesivo de azúcar en Colombia (Archivo de video), 12 de septiembre de 2016. Disponible en: https://www.youtube.com/ watch?v=3wBWkMcBdkY\&feature=youtu.be

16. RedPapaz, No comas más mentiras (Mensaje en Blog), Noviembre de 2017. [citado 22 de febrero de 2019]. Disponible en: https:// www.nocomasmasmentiras.org/nuestro_mensaje/.

17. RedPapaz. Insistencia selección de expediente de Tutela T-7139620, Febrero de 2019 [Internet]. [citado 22 de febrero de 2019]. Recuperado de http://www.corteconstitucional.gov.co/ secretaria/insistencias/Insistencias \% 20Auto \% 20del $\% 2028 \% 20$ de $\% 20$ enero $\% 20$ de $\% 202019 \% 20$ Notificado $\% 20$ el $\% 2011 \% 20$ de $\% 20$ febrerode $\% 202019 \% 20$ con $\% 20$ vencimiento $\% 20$ el $\% 20$ $26 \% 20$ de $\% 20$ febrero $\% 20$ de $\% 202019 /$ T7 $139620 \%$ 20DRA. $\% 20$ FAJARDO.pdf

18. Instituto Nacional de Vigilancia de Medicamentos - Invima, Dirección de Responsabilidad Sanitaria (29 de octubre de 2018). Auto No. 2018012992, Proceso 201601561; 2018. [citado 22 de febrero de 2019]. Disponible en https://www.invima.gov.co/ avisos-direcci \% C3\% B3n-de-responsabilidad-sanitaria-2018. html? start $=22$

19. Piñeros Ospina, Carolina. Solicitud ante la Corte Constitución para revision expediente T-7139620 [Pdf File]. Corporación Colombiana de Padres y Madres - Red PaPaz: 2019. [citado 22 de febrero de 2019]. Disponible en: https://cdn.dejusticia.org/wpcontent/uploads/2019/01/Solicitud-de-Revisi\%C3\%B3n-CorteConstitucional-Tutela-Red-Papaz.pdf

20. Dejusticia. Intervención del Centro de Estudios de Derecho, Justicia y Sociedad -Dejusticia- en el proceso de tutela T-7.139.620[Internet]; 2019. Disponibleen:https://www.dejusticia. org/wp-content/uploads/2019/05/Intervencio\% CC\% 81n-Hit-yFruper_CorteConstitucional.pdf

21. Resolución 19022 de 31 de mayo de 2019 por la cual se inicia una investigación administrativa mediante formulación de cargos (2019).

22. Cámara de Representantes. Proyecto de Ley 214 de 2018, "por medio de la cual se promueve el acceso a información necesaria para fomentar entornos alimentarios saludables y prevenir Enfermedades No Transmisibles y se adoptan otras disposiciones".
Gaceta del Congreso. Año XXVII, No. 883, 23 de octubre de 2018.

23. Blecher E, Liber AC, Drope JM, Nguyen B, Stoklosa M. Peer Reviewed: Global Trends in the Affordability of Sugar-Sweetened Beverages, 1990-2016. Prev Chronic Dis. 2017;14:E37.

24. Rebholz CM, Young BA, Katz R, Tucker KL, Carithers TC, Norwood AF, Correa A. Patterns of beverages consumed and risk of incident kidney disease. Clin J Am Soc Nephrol. 2019;14(1):49-56.

25. Caravalí-Meza NY, Jiménez-Cruz A, Bacardí-Gascón M. Estudio prospectivo sobre el efecto del consumo de bebidas azucaradas sobre la obesidad en un periodo de 12 meses en mexicanos de 15 a 19 años. Nutrición Hospitalaria. 2016;33(2):270-6.

26. World Health Organization. Fiscal policies for diet and prevention of noncommunicable diseases: technical meeting report, 5-6 May 2015, Geneva, Switzerland: World Health Organization; 2016.

27. Superintendencia de Industria y Comercio. Superindustria ordena retirar comercial de TV sobre supuestos efectos nocivos del consumo de bebidas azucaradas. [citado 01 de diciembre 2017] Disponible en: http://www.sic.gov.co/noticias/superindustriaordena-retirar-comercial-de-tv-sobre-supuestos-efectos-nocivosdel-consumo-de-bebidas-azucaradas.

28. Corte Constitucional. Sentencia de tutela T - 543 de 2017. [MP. Diana Fajardo Rivera] [Internet]. 25 de agosto de 2017. [citado 15 de noviembre de 2017]. Disponible en: http://www. corteconstitucional.gov.co/relatoria/2017/t-543-17.htm

29. Manso, T.H. Del consumidor informado al consumidor real. El futuro del Derecho de Consumo europeo. Dykinson; 2016

30. Hernández Díaz-Hambrona, M.D. Consumidor vulnerable. Madrid, España: Editorial Reus; 2015.

31. Urquijo BI, Sayas CR. El consumidor alimentario vulnerable en Colombia: Un análisis desde la normativa europea. Revista de Derecho Privado. 2016;(56):1-20.

32. Gesualdo N, Yanovitzky I. Advertising Susceptibility and Youth Preference for and Consumption of Sugar-Sweetened Beverages: Findings from a National Survey. J Nutr Educ Behav. 2019;51(1):1622.

33. Rivera JÁ, de Cossío TG, Pedraza LS, Aburto TC, Sánchez TG, Martorell R. Childhood and adolescent overweight and obesity in Latin America: a systematic review. Lancet Diabetes Endocrinol. 2014;2(4):321-32.

34. Ballesteros Arribas JM, Dal-Re Saavedra M, Pérez-Farinós N, Villar Villalba C. La estrategia para la nutrición, actividad física y prevención de la obesidad: estrategia NAOS. Rev Esp Salud Pública. 2007;81:443-9.

35. Andreyeva T, Chaloupka FJ, Brownell KD. Estimating the potential of taxes on sugar-sweetened beverages to reduce consumption and generate revenue. Preventive medicine. 2011;52(6):413-6.

36. Jou J, Techakehakij W. International application of sugarsweetened beverage (SSB) taxation in obesity reduction: factors that may influence policy effectiveness in country-specific contexts. Health Policy. 2012;107(1):83-90.

37. Thomas-Meyer M, Mytton O, Adams J. Public responses to proposals for a tax on sugar-sweetened beverages: a thematic analysis of online reader comments posted on major UK news websites. PloS one. 2017 Nov 22;12(11):e0186750. 
38. Popkin BM, Hawkes C. Sweetening of the global diet, particularly beverages: patterns, trends, and policy responses. Lancet Diabetes Eendocrinol. 2016 Feb 1;4(2):174-86.

39. Ortún V, López-Valcárcel BG, Pinilla J. El impuesto sobre bebidas azucaradas en España. Rev Esp Salud Pública. 2017;90:e20007.

40. Lal A, Mantilla-Herrera AM, Veerman L, Backholer K, Sacks G, Moodie M, Siahpush M, Carter R, Peeters A. Modelled health benefits of a sugar-sweetened beverage tax across different socioeconomic groups in Australia: A cost-effectiveness and equity analysis. PLoS Medicine. 2017;14(6):e1002326.

41. Von Tigerstrom B. Taxing sugar-sweetened beverages for public health: legal and policy issues in Canada. Alta. L. Alberta Law Review. 2012;50:37.

42. Heise TL, Katikireddi SV, Pega F, Gartlehner G, Fenton C, Griebler U, Sommer I, Pfinder M, Lhachimi SK. Taxation of sugar-sweetened beverages for reducing their consumption and preventing obesity or other adverse health outcomes. Cochrane Database of Systematic Reviews. 2016(8).

43. Organización Panamericana de la Salud. Experiencia de México en el establecimiento de impuestos a las bebidas azucaradas como estrategia de salud pública [Internet]. México: OPS, Representación de México; 2015. [citado 22 de febrero de 2019] Disponible en: http://iris.paho.org/xmlui/bitstream/ handle/123456789/18390/978-92-75-31871-3_esp.pdf

44. Paraje G. The effect of price and socio-economic level on the consumption of sugar-sweetened beverages (SSB): the case of Ecuador. PloS one. 2016 Mar 30;11(3):e0152260.

45. Cámara de Representantes. Proyecto de Ley por medio de la cual se adopta una reforma tributaria estructural, se fortalecen los mecanismos para la lucha contra la evasión y la elusión fiscal, y se dictan otras disposiciones.[Proyecto de Ley 178/16]. Gaceta del Congreso 894/16; 19 de octubre de 2016.

46. Cadena Gaona E, Rodríguez A, Araque A, Londoño M, Franco Restrepo C, Zapata Jaramillo $\mathrm{T}$, et al. Impuesto a las bebidas azucaradas [Internet]. Papeles En Salud 15; 2016. [citado 22 de febrero de 2019]. Recuperado de: https://www.minsalud.gov.co/ sites/rid/Lists/BibliotecaDigital/RIDE/DE/AS/papeles-salud-n5. pdf

47. Dejustica. Marco legal del impuesto a las bebidas azucaradas en Colombia [Internet]. Bogotá; 2017. [16 de enero de 2018]. Recuperado en: https://www.dejusticia.org/wp-content/ uploads/2017/04/fi_name_recurso_868.pdf

48. Vlex Colombia. Comentarios al Proyecto de Ley 163 de 2016 Senado (PL 178 de 2016 Cámara) [Internet]. Colombia; Vlex Colombia; 2016. [citado 22 de febrero de 2019] Disponible en: https://vlex. com.co/vid/comentarios-proyecto-ley-163-655799829

49. Cámara de Representantes, Informe de Ponencia Primer Debate Proyecto de Ley 178 de 2016 Cámara (PL 163 de 2016 Senado) [Internet]. Gaceta del Congreso 1090/16. [citado 22 de febrero de 2019]. Recuperado en: https://vlex.com.co/vid/comentariosproyecto-ley-163-655799829

50. Rodríguez L., Alejandro. Ese tal impuesto a las bebidas azucaradas no existe. Dejusticia: derecho, justicia, sociedad [Internet]. (citado febrero 18 de 2019). Recuperado de: https://www.dejusticia.org/ column/ese-tal-impuesto-a-las-bebidas-azucaradas-no-existe/

51. Cámara de Representantes. Proyecto de Ley 007 de 2016 Por medio de la cual se establecen normas sobre la información nutricional, el etiquetado de las bebidas azucaradas y se dictan otras disposiciones. - Ley para el Consumo Informado del Azúcar. 2016 [Internet]. Colombia. [citado 22 de febrero de 2019]. Recuperado de:http://leyes.senado.gov.co/proyectos/index.php/textosradicados-senado/pl-2016-2017/660-proyecto-de-ley-007-de-2016

52. Cámara de Representantes. Proyecto de ley por medio de la cual se establecen medidas de salud pública para el control de la obesidad y otras Enfermedades Crónicas no Transmisibles derivadas y se dictan otras disposiciones [Internet]. Colombia: Congreso visible Universidad de los Andes; 2017. [citado 22 de febrero de 2019]. Recuperado de: https://www.asivamosensalud.org/politicaspublicas/normatividad-proyectos-de-ley/salud-publica/proyectode-ley-019-de-2017-medidas

53. Proyecto de Ley 214 de 2018, "por medio de la cual se promueve el acceso a información necesaria para fomentar entornos alimentarios saludables y prevenir Enfermedades No Transmisibles y se adoptan otras disposiciones. Gaceta del Congreso: Senado y Cámara 2018;XXVII(883):1-32.

54. Roberto CA, Wong D, Musicus A, Hammond D. The influence of sugar-sweetened beverage health warning labels on parents' choices. Pediatrics. 2016;137(2):e20153185.

55. Gómez LF, Ibarra ML, Lucumí DI, Arango CM, Parra A, Cadena Y, Erazo V, Parra DC. Alimentación no saludable, inactividad física y obesidad en la población infantil colombiana: un llamado urgente al estado y la sociedad civil para emprender acciones efectivas. Global Health Promotion. 2012;19(3):87-92.

56. International Covenant on Economic, Social and Cultural Rights. Adopted and opened for signature, ratification and accession by General Assembly resolution 2200A (XXI) of 16 December 1966 [Internet]. UN General Assembly; 1976 [citado 201922 de febrero]; Recuperado de: https://www.refworld.org/docid/3ae6b36c0. html.

57. Organización de las Naciones Unidas. Informe del Relator Especial sobre el derecho de todapersona al disfrute del más alto nivel posible de saludfísica y mental, Anand Grover. Los alimentos poco saludables, las enfermedades no transmisiblesy el derecho a la salud. Organización de las Naciones Unidas; 2014. p. 27.

58. World Health Organization. Guideline: sugars intake for adults and children. Geneva, Switzerland: World Health Organization; 2015.

59. World Health Organization. Taxes on sugary drinks: Why do it? Geneva, Switzerland: World Health Organization: 2016.

60. Caro JC, Ng SW, Bonilla R, Tovar J, Popkin BM. Sugary drinks taxation, projected consumption and fiscal revenues in Colombia: Evidence from a QUAIDS model. PloS one. 2017;12(12):e0189026.

61. Weaver D, Finke M. The relationship between the use of sugar content information on nutrition labels and the consumption of added sugars. Food Policy. 2003;28(3):213-9.

62. De Lecuona, I. Informació alimentària: qüestions ètiques, jurídiques i polítiques. Barcelona: Edicions Universitat Barcelona; 2017. 